\title{
Seasonal ataxia in western Nigeria: evaluation of the impact of health education on hospital prevalence
}

Bolanle Adamolekun

\begin{abstract}
Study objective - The study aimed to evaluate the impact of focused health education on the hospital prevalence of a seasonal ataxic syndrome which occurs exclusively in people of low income after eating the larvae of Anaphe venata Butler (Lepidoptera, Notodontidae).

Design - Retrospective impact evaluation study.

Setting - Wesley Guild Hospital, Ilesha, western Nigeria from where the syndrome was first described.

Methods-Hospital workers were informed of recent research findings on the aetiological role of Anaphe venata larvae consumption in the annual epidemics of seasonal ataxia. They were encouraged to educate hospital patients and their relatives and to ask them to pass this information to neighbours and other family members who are usually of the same social strata. The number of admissions for seasonal ataxia in the pre-intervention years (1990 and 1991) and in the intervention years (1992 and 1993) were extracted from records. The toal number of casualty admissions between July and November of each year were also obtained. The period prevalence rates of the disease in each year of the study period were calculated and compared.
\end{abstract}

Results - The pre-intervention prevalence rates were $1 \cdot 27 \%$ and $1.59 \%$ for 1990 and 1991 respectively. The prevalence rates in the intervention years of 1992 and 1993 were $0.58 \%$ and $0.006 \%$, representing a $65.5 \%$ reduction between 1991 and 1992, and a 99\% reduction between 1992 and 1993.

Conclusion - The health education technique used is an inexpensive but effective way of providing the intervention needed for the control of nutrition related diseases in the poor, largely illiterate segment of communities that are most vulnerable.

( $\mathcal{F}$ Epidemiol Community Health 1995;49:489-491)

Epidemics of a seasonal ataxic syndrome occur annually in the rainy season in parts of western Nigeria. This disease is characterised by intention tremors, cerebellar ataxia, nystagmus, and varying levels of impaired consciousness. ${ }^{12}$
The syndrome was highly prevalent in the Ijesha people living in and around Ilesha, a town in the northern half of the rain forest zone of western Nigeria and it was from here that the syndrome was first described. ${ }^{1}$

Seasonal ataxia remained an annually recurring enigma of unknown aetiology until 1991 when acute thiamine deficiency was proposed as the mechanism. It was suggested that the ataxia occurred in poor people who were marginally deficient in thiamine because of a limited diet of high carbohydrate meals containing thiamine-binding cyanogenetic glycosides, and who suffered a seasonal exacerbation of their thiamine deficiency from thiaminases present in seasonal foods peculiar to the area of endemicity. ${ }^{3}$

Epidemiological studies begun in 1991 confirmed that the syndrome occurred exclusively in poor people subsisting almost entirely on a monotonous high carbohydrate diet and arose hours after a meal of stew containing the larvae of Anaphe venata Butler (Lepidoptera, Notodontidae). ${ }^{45}$ The latter is a seasonal, protein food peculiar to the area of endemicity and whose period of availability in the markets had always coincided with the period of seasonal occurrence of the ataxic syndrome. This seasonal larval food may well contain the thiaminases or antithiamines thought ${ }^{3}$ to precipitate a seasonal exacerbation of acute thiamine deficiency. Confirmation of the role of thiamine deficiency in the aetiology of the seasonal ataxia was also obtained from the results of a double blind, controlled trial showing the efficacy of thiamine hydrochloride treatment. ${ }^{6}$

With the implication of the larvae of Anaphe venata in the aetiology of the disease and the identification of the susceptible stratum of the population, control and eradication of the seasonal epidemics of ataxia became feasible.

There was, however, no ready access to funds to undertake a formal community based health education programme. The poor, largely illiterate segment of the population that was most susceptible to the disease was also judged to have little access to information disseminated by radio, television, or newspapers.

A hospital based, focused health education programme to reach the susceptible segment of the society was devised. An evaluation of the impact of this health education programme on the hospital prevalences of the seasonal ataxia is now reported. 
Impact of health education intervention (from 1992) on the hospital prevalences of seasonal ataxia at the Wesley Guild hospital, Ilesha, Nigeria

\begin{tabular}{lcccc}
\hline & 1990 & 1991 & 1992 & 1993 \\
\hline Total no of admissions (July-November) & 3776 & 3510 & 3594 & 3239 \\
No of admissions for seasonal ataxia & 48 & 56 & 21 & 2 \\
Period prevalence rate (\%) & 1.27 & 1.59 & 0.58 & 0.006
\end{tabular}

\section{Methods}

HEALTH EDUCATION

The focused health education programme, which started in July 1992 and continued through the 1992 and 1993 disease seasons, consisted of one hospital seminar, ward seminars, and informal talks to apprise nurses and doctors in the medical wards and casualty of the disease, particularly about the recent implication of Anaphe venata larvae consumption in its aetiology.

These seminars and talks were given by the author, using slides. The hospital seminar was organised by the hospital management, who had shown considerable interest in clarifying the aetiology of the seasonal ataxia. Previous aetiological hypotheses of viral encephalitis ${ }^{1}$ and of toxins in new yam ${ }^{2}$ were reviewed and epidemiological evidence against them were presented. The recent studies implicating acute thiamine deficiency from Anaphe venata consumption were also presented. There was a period of general discussion during which enquiries relating to the recent studies were answered.

Ward seminars and talks followed a similar format.

The doctors and nurses were encouraged to educate patients admitted to hospital with the disease, as well as their visiting relatives, about the risks of eating Anaphe venata. The health message was that Anaphe venata consumption caused the ataxic symptoms in persons who were susceptible because of their inadequate nutrition. The patients were advised not to eat it since their present illness had shown that they and probably their relatives had a high risk of developing the disease in future epidemics. It was expected that the patients and their relatives would pass this information on to their neighbours and other members of their family, who are usually of the same social strata.

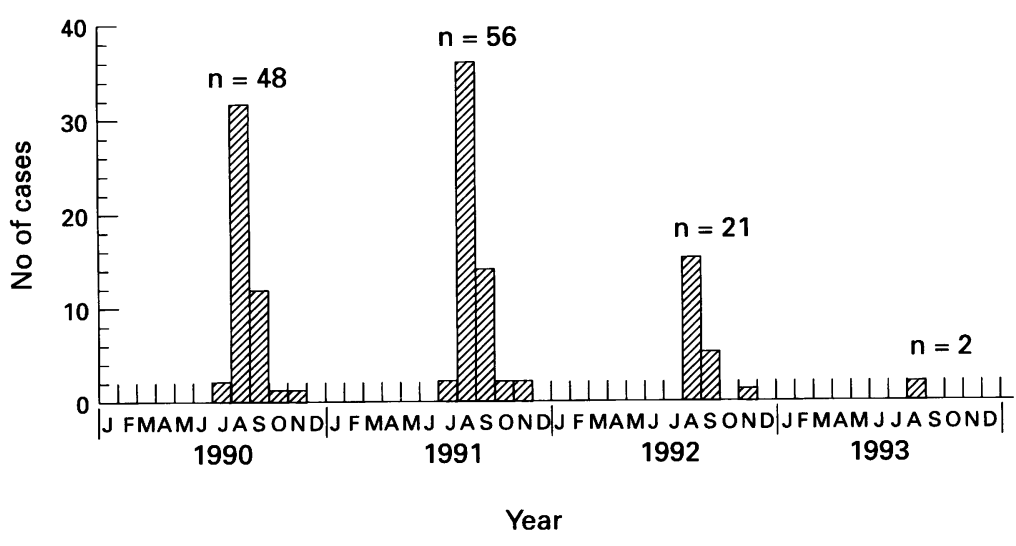

Number of cases of seasonal ataxia between 1990 and 1993.
IMPACT EVALUATION

The decision to undertake this evaluation was taken at the end of November 1993. The case notes and nursing records of all patients admitted to the casualty department at the Wesley Guild hospital, Ilesha were consulted to determine the number of admissions for seasonal ataxia in the pre-intervention years of 1990 and 1991, and in the intervention years of 1992 and 1993. The total number of casualty admissions between July and November of each year were also obtained. The period prevalence rates of the disease in each year of the study period were calculated and compared. Casualty records were preferred to medical ward records because patients with seasonal ataxia were often discharged home from casualty without admission to the wards. Furthermore, the admission policy of the teaching hospital allows for admission of patients into casualty without payment of an admission deposit, while such deposits are mandatory for ward admissions. Acutely ill but impecunious patients therefore have access to casualty care.

\section{Results}

The health talks were enthusiastically received by the health workers, many of whom had apparently had some doubts about the previous aetiological suggestions. Many of the health workers were eager to confirm the finding of Anaphe venata consumption in their patients with the seasonal ataxia, and on confirming this proceeded to give the health talk.

The patients' and relatives' acceptance of the health talks were probably reinforced by their noticing that other patients with similar symptoms also admitted to having eaten the Anaphe venata larvae.

The figure shows the numbers of cases between 1990 and 1993. The prevalence rates of seasonal ataxia between July and November at the Wesley Guild hospital, Ilesha before (1990, 1991) and with health education intervention $(1992,1993)$ are shown in table 1 . The prevalence rates in 1990 and 1991 were $1.27 \%$ and $1.59 \%$ respectively. The prevalence rates in 1992 and 1993 were $0.58 \%$ and $0.006 \%$. These rates represent a $65.5 \%$ reduction in prevalence rates between 1991 and 1992, and a $99 \%$ reduction between 1992 and 1993. The distribution of cases of the ataxic syndrome by month in the years of study confirm its seasonality, with a consistent peak in August (figure).

\section{Discussion}

The striking reduction in the hospital prevalence rates of seasonal ataxia observed in this study coincided with, and is probably the result of, starting a health education programme directed at hospital workers, admitted patients and their relatives concerning the aetiological association of the disease with Anaphe venata consumption.

It seems unlikely that the lower prevalence is the result of a spontaneous reduction in the disease, as such a decline had never previously 
occurred. ${ }^{12}$ Indeed, in 1993 when a hospital prevalence of $0.006 \%$ was obtained in this study, a community prevalence of $1 \cdot 87 \%$ was obtained in an epidemic of the disease in Ikare town (population 60000 ), $150 \mathrm{~km}$ away. ${ }^{7}$

Since people eating a low protein diet are selectively susceptible to the seasonal ataxic syndrome, it would seem theoretically possible to eradicate the disorder by improving the protein nutrition of people in the area of endemicity. ${ }^{8}$ However, there is no evidence that protein nutrition in western Nigeria has improved enough since 1991 to account for the dramatic decline observed. On the contrary, there has been a progressive economic deterioration during this period; with the resulting increased cost of protein foods leading to inadequate protein nutrition and an increased pool of subjects susceptible to the seasonal ataxia.

The long standing, widespread use of the Anaphe venata larvae for food seems justified by studies indicating that the nutritional value of the larvae is comparable to that of chicken eggs. ${ }^{9}$ Indeed, mass rearing of the larva as an alternative protein source had been advocated. ${ }^{9}$ A wholesale campaign against its sale and consumption by everyone might cause panic, and deprive a large number of people of an important seasonal dietary protein source.

One possible control strategy is the therapeutic supplementation of diets in the endemic areas with thiamine-containing preparations to boost the pre-morbid thiamine status of individuals consuming the larvae. We are presently planning a community based, controlled study of multivitamin supplementation to evaluate the acceptability and effectiveness of this control strategy.

The health education technique used in this study allows an oral information cascade from the health workers through patients and relatives to the susceptible segment of the society.
This oral route of information dissemination accords with the oral tradition of many communities in developing countries, and is therefore readily accepted. It is also economical, and does not require any technology. The benign, non-fatal course of the seasonal ataxia ${ }^{12}$ makes it particularly suitable for the focused health education intervention used. Epidemic diseases with fatalities will require more urgent intervention.

With the deterioration in the economies of many developing countries and the consequent increase in the cost of protein foods, diseases of malnutrition, such as the seasonal ataxic syndrome, may emerge as a major public health problem. The focused health education technique used in this report may be an inexpensive but effective way of providing intervention for the control of nutritional diseases in the indigent, largely illiterate segment of communities that are most vulnerable. It may also be useful in other non-fatal diseases of different epidemiology that occur in rural communities.

1 Wright J, Morley DC. Encephalitis tremens. Lancet 1958;i: $871-3$.

2 Osuntokun BO. Epidemic ataxia in Western Nigeria. BMF 1972;ii:589.

3 Adamolekun B. A seasonal ataxic syndrome in South-Western Nigeria: An etiological hypothesis of acute thiamine deficiency. Ethnic Dis 1992;2(2):185-6.

4 Adamolekun B. Anaphe venata entomophagy and seasonal ataxic syndrome in Southwest Nigeria. Lancet 1993;341: 629.

5 Adamolekun B. 1993 Bruce Schoenberg International award and Lecture in Neuroepidemiology: Epidemiological studies of the etiology of a seasonal ataxia in Nigerians (abstract). Neurology 1993;43:1419.

6 Adamolekun B, Adamolekun WE, Sonibare AD, Sofowora $\mathrm{G}$. A double-blind, placebo-controlled study of the efficacy of thiamine hydrochloride in a seasonal ataxia in Nigerians. Neurology 1994;44:549-51.

7 Adamolekun B, Ibikunle FR. Investigation of an epidemic of seasonal ataxia in Ikare, western Nigeria. Acta Neurol Scand 1994;90(5):309-311.

8 Adamolekun B, Lawal O, Ndububa DA, Balogun MO. Serum albumin levels and intra-ethnic variations in susceptibility to a seasonal ataxia in Nigerians. Ethnic Dis 1995; (in press).

9 Ashiru MO. The food value of the larvae of Anaphe venata Butler (Lepidoptera: Notodontidae). Ecology of Food and
Nutrition 1988;22:313-20. 